Supplementary information

\title{
Complete characterization of polyacyltrehaloses from Mycobacterium
}

tuberculosis H37Rv biofilm cultures by multiple-stage linear ion-trap mass spectrometry reveals a new tetraacyltrehalose family

Georgiana E Purdy ${ }^{\dagger}$ and $*$ Fong-Fu Hsu ${ }^{\ddagger}$

${ }^{\dagger}$ Department of Microbiology and Immunology, Oregon Health \& Science University, Portland, OR 97239, USA

†Mass Spectrometry Resource, Division of Endocrinology, Diabetes, Metabolism, and Lipid

Research, Department of Internal Medicine, Washington University School of Medicine, St.

Louis, MO 63110 USA

*To whom the correspondence should be addressed: Dr. Fong-Fu Hsu, Box 8127, Washington

University School of Medicine, 660 S Euclid, St. Louis, MO 63110. Tel: 314-362-0056, e-mail:

fhsu@im.wustl.edu. 
ESI LC/MS analysis:

LC/MS analysis was carried out by an Agilent 1290 HPLC connected to an Agilent 6550 QTOF mass spectrometer controlled by Mass Hunter software. Separation of Mycobacterium tuberculosis H37Rv biofilm lipid was achieved by a Supelco $100 \times 2.1 \mathrm{~mm}(2.7 \mu$ particle size $)$ Ascentis C-8 column at a flow rate of $250 \mu \mathrm{L} / \mathrm{min}$. The mobile phase contained $5 \mathrm{mM}$ ammonium formate ( $\mathrm{pH}$ 5.0) in both solvent A-acetonitrile:water (60:40, v/v) and solvent Bisopropanol:acetonitrile (90:10, v/v). A gradient elution in the following manner was applied: 68\% A, 0-3 min; 68-50\% A, 3-8 min; 50-35\% A, 8-13 min; 35-25\% A, 13-18 min; 25-15\% A, 18-28 min; 15-5\% A, 28-33 min; 5-0\% A, 33-40 min; 0\% A, 40-50 min; 0-68\% A, 50-60 min. Two separate positive-ion and negative-ion ESI LC/MS analyses were performed, and around $20 \mu \mathrm{g}$ in $20 \mu \mathrm{L} \mathrm{CHCl}_{3} / \mathrm{CH}_{3} \mathrm{OH}(2 / 1$, v/v) of lipid extract were injected in each analysis.

The HPLC retention time and the positive ion (seen as the $\left[\mathrm{M}+\mathrm{NH}_{4}{ }^{+}\right]$ion; top panel) and negative ion (seen as the $\left[\mathrm{M}+\mathrm{HCO}_{2}^{-}\right]$ion; bottom panel) ESI mass spectra of the polyacyl trehalose subfamilies including DAT (Fig S1a), TAT (Fig S1b), TetraAT (Fig S1c), and PAT (Fig S1d) are shown below:

a. DAT (elution time: 20.9-22.9 min) (Fig S1a)

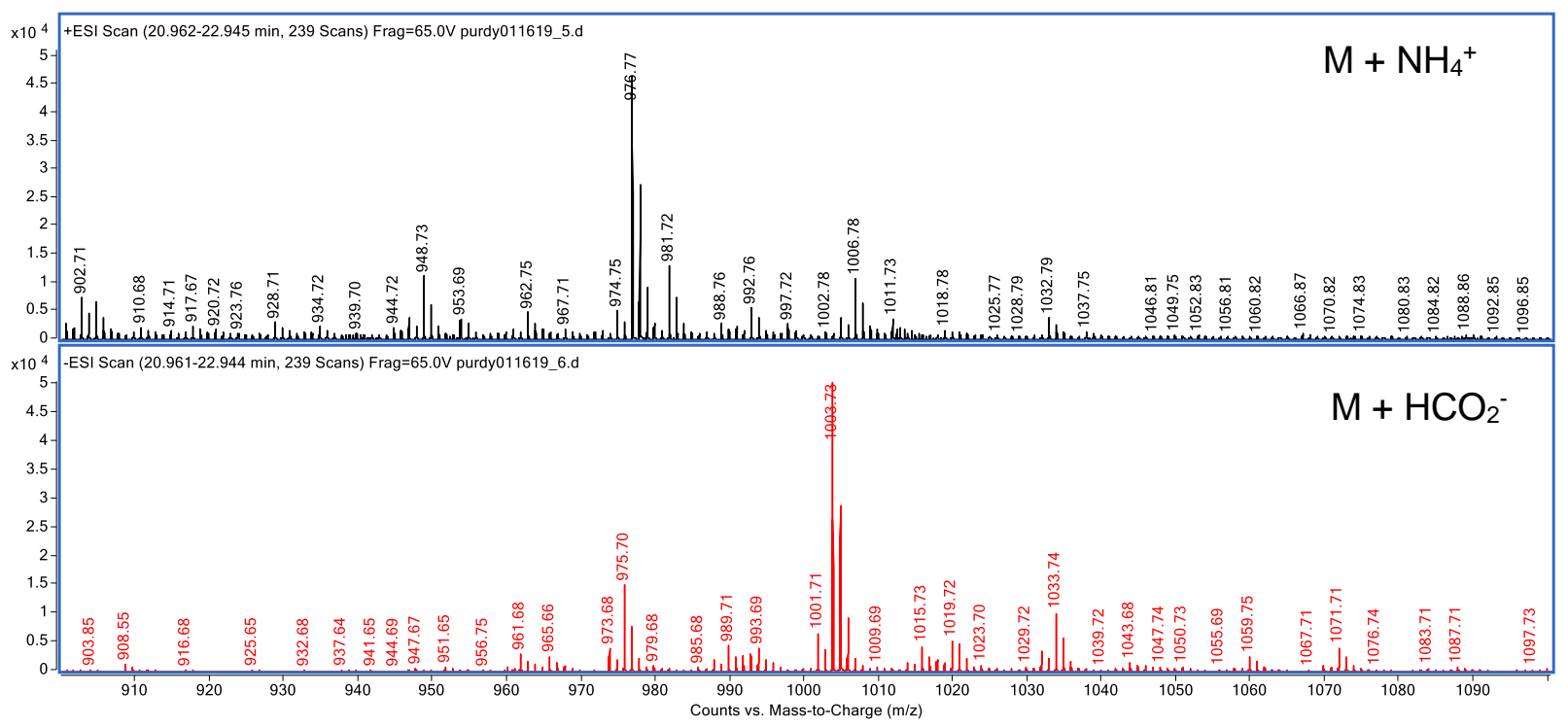


b. TAT (elution time: 30.9-32.2 min) (Fig S1b)

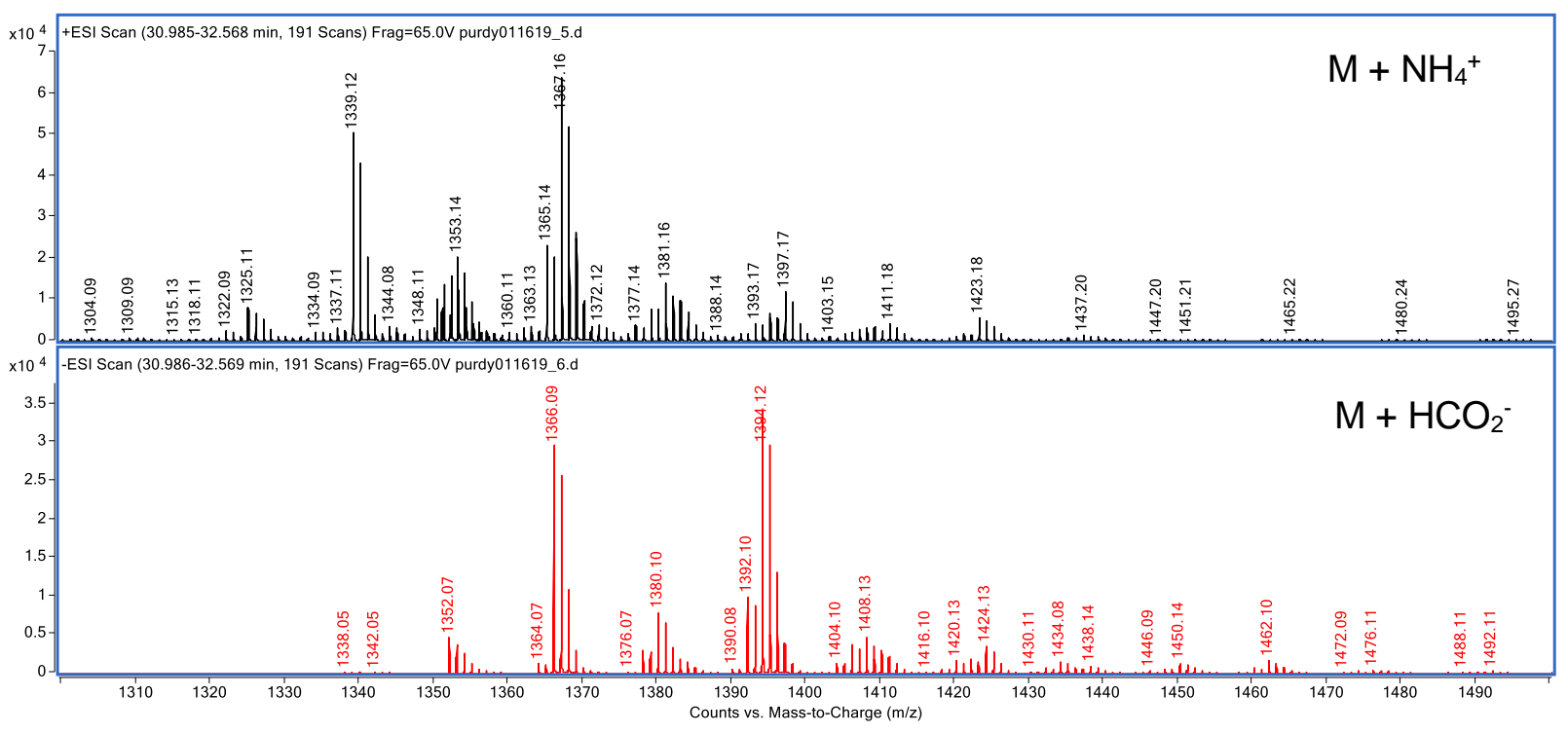

c. TetraAT (elution time: $34.3-36.5 \mathrm{~min}$ ) (Fig S1c)

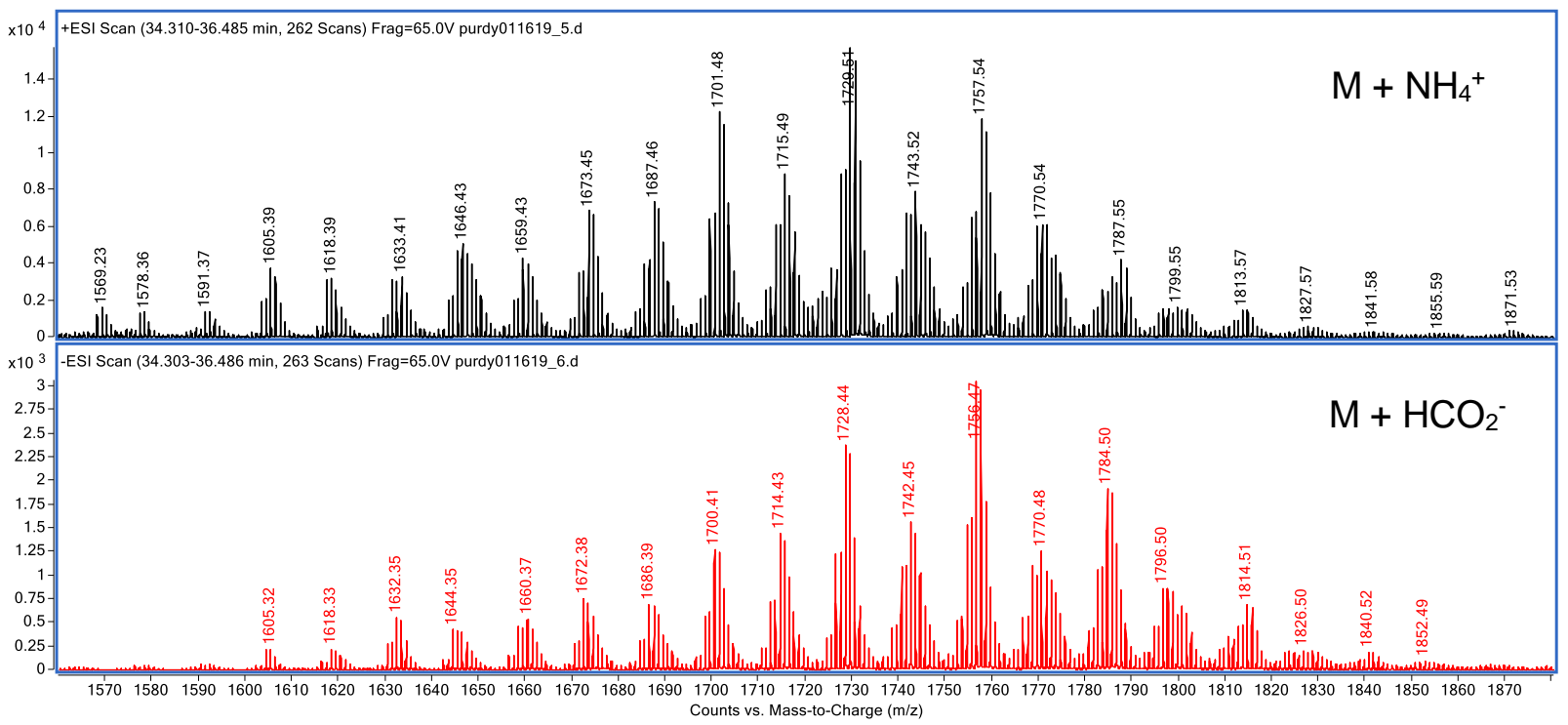


d. PAT (elution time: 37.5-39.6 min) (Fig S1d)

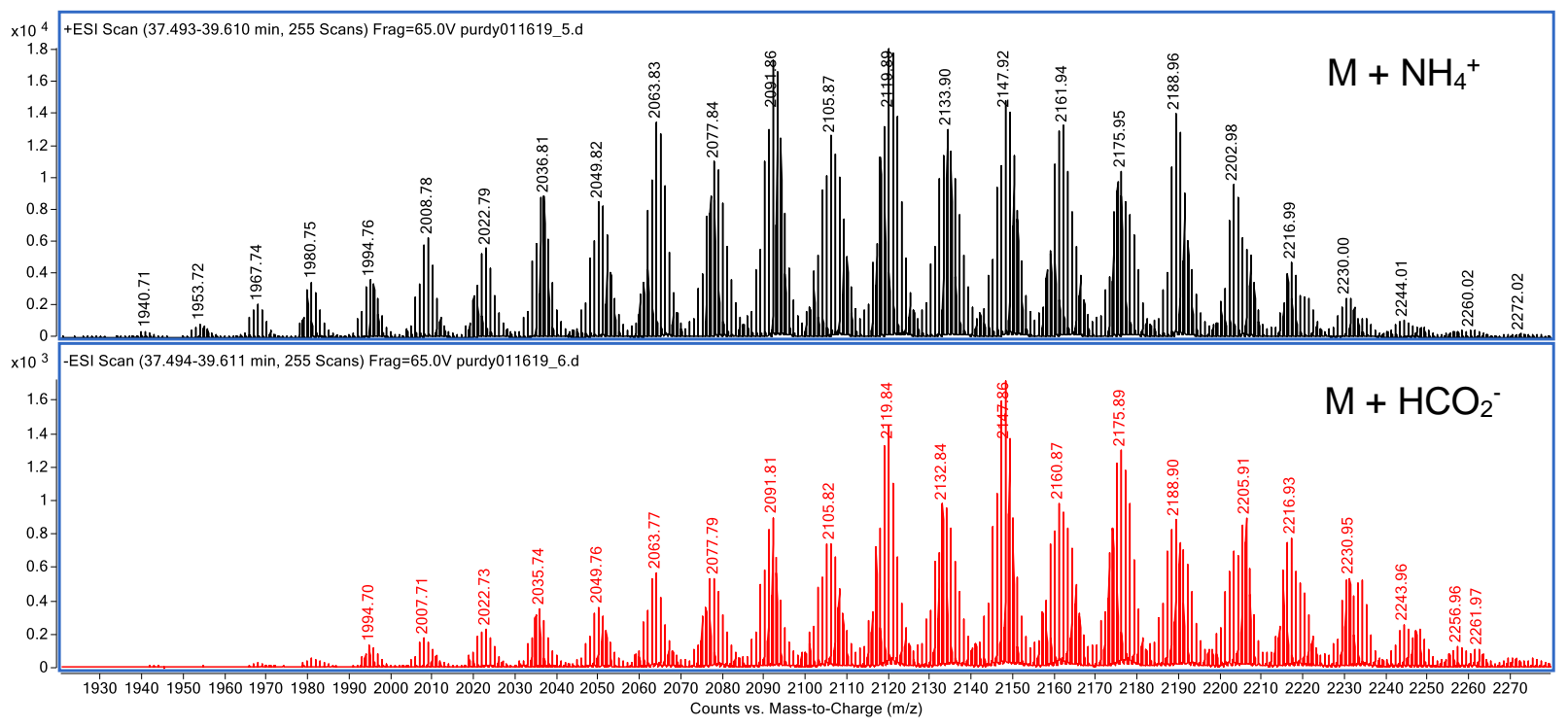

Figure S1. The ESI mass spectra of the polyacyl trehalose subfamilies including (a) DAT (elution time: 20.9-22.9 min), (b) TAT (elution time: 30.9-32.2 min), (c) TetraAT (elution time: 34.3-36.5 min), and (d) PAT (elution time: 37.5-39.6 min). Please note that in each of the spectra representing the glycolipid subfamilies, the top panels show the [M $\left.+\mathrm{NH}_{4}\right]^{+}$ions obtained in the positive ion mode, and the bottom panels represent the $[\mathrm{M}+$ $\left.\mathrm{HCO}_{2}\right]^{-}$ions obtained in the negative ion mode. The positive/negative ion ESI/MS (top/bottom) profiles for each subfamily are nearly identical, leading to confirm the molecular species. 


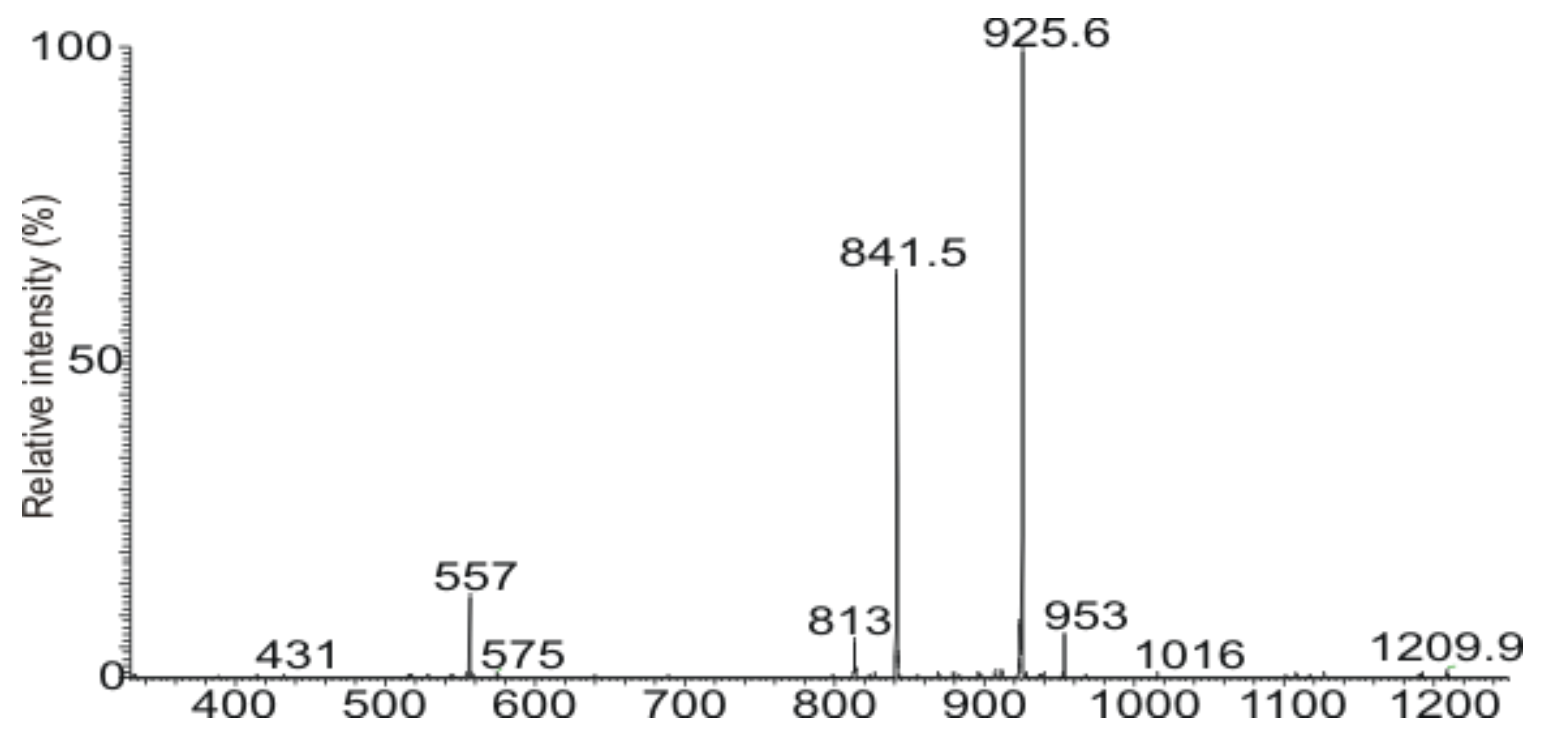

Figure S2. The LIT MS ${ }^{3}$ spectrum of the ion of $1209.9(2152 \rightarrow 1209.9)$ arising from the $[\mathrm{M}$ $+\mathrm{Na}]^{+}$ion of $(18: 0 / 24: 0 / 27: 1)(27: 1 / 27: 1)-\mathrm{PAT}$ at $\mathrm{m} / \mathrm{z} 2152$. This spectrum is identical to the $\mathrm{MS}^{3}$ spectrum of the ion of $1209.9(1372.1 \rightarrow 1209.9)$ from 18:0/24:0/27:1-TAT (Fig.5b) and the $\mathrm{MS}^{3}$ spectrum of the $\mathrm{m} / \mathrm{z} 1209.9(1762 \rightarrow 1209.9)$ ion originated from the $[\mathrm{M}+\mathrm{Na}]^{+}$ion of $(18: 0 / 24: 0 / 27: 1)(27: 1)-T e t r a A T$ at $\mathrm{m} / \mathrm{z}$ 1762.4. 
100
50

0

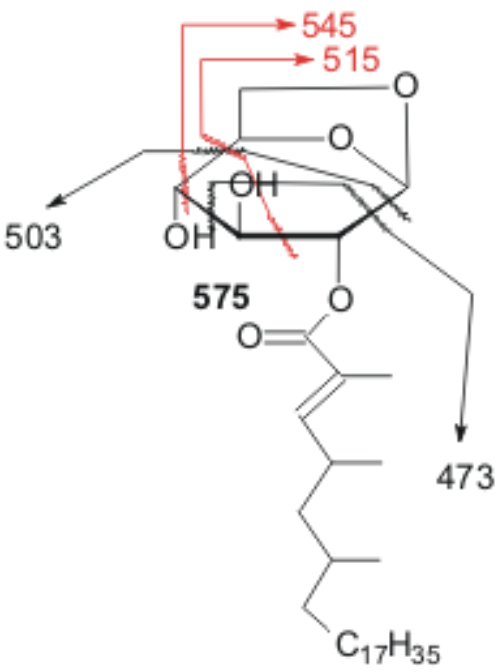

557

章

$731 \quad 503$

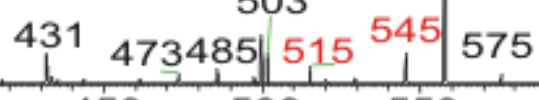

$\mathrm{m} / \mathrm{z}$

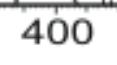

450

500

Figure S3. The LIT MS ${ }^{3}$ spectrum of the ion of $\mathrm{m} / \mathrm{z} 575(1762 \rightarrow 593 \rightarrow 575)$ originated from the $[\mathrm{M}+\mathrm{Na}]^{+}$ion of $(18: 0 / 24: 0 / 27: 1)(27: 1)-$ TetraAT at $\mathrm{m} / \mathrm{z}$ 1762. This $\mathrm{MS}^{3}$ spectrum contained fragment ions pointing to the location of the 27:1-FA substituent at C2' (of Glc2) (see inset for the fragmentation scheme). 


\begin{tabular}{|c|c|c|c|c|c|}
\hline $\begin{array}{l}\text { measured } \\
\mathrm{m} / \mathrm{z} \text { (Da) }\end{array}$ & $\begin{array}{r}\text { Theo. Mass } \\
\text { Da }\end{array}$ & $\begin{array}{l}\text { Delta (mmu) } \\
\mathrm{mDa}\end{array}$ & $\begin{array}{l}\text { Relative } \\
\%\end{array}$ & RDB equiv. & Composition \\
\hline 168.0809 & 168.0807 & 0.12 & 1.83 & 8.5 & $\mathrm{C} 12 \mathrm{H} 10 \mathrm{~N}$ \\
\hline 169.0886 & 169.0886 & 0 & 82.31 & 8 & $\mathrm{C} 11 \mathrm{H} 12 \mathrm{~N}$ \\
\hline 183.0917 & 183.0917 & 0.05 & 100 & 8.5 & C12 H11 N2 \\
\hline 211.0867 & 211.0866 & 0.16 & 2.1 & 9.5 & C13 H11 O N2 \\
\hline 223.1231 & 223.1230 & 0.16 & 6.22 & 9.5 & C15 H15 N2 \\
\hline 251.118 & 251.1179 & 0.15 & 14.58 & 10.5 & C16 H15 O N2 \\
\hline 265.1337 & 265.1335 & 0.11 & 6.79 & 10.5 & C17 H17 O N2 \\
\hline 279.1493 & 279.1492 & 0.06 & 41.85 & 10.5 & C18 H19 O N2 \\
\hline 293.165 & 293.1648 & 0.12 & 1.88 & 10.5 & C19 H21 O N2 \\
\hline 307.1805 & 307.1805 & 0.04 & 0.3 & 10.5 & $\mathrm{C} 20 \mathrm{H} 23 \mathrm{O} \mathrm{N} 2$ \\
\hline 321.1961 & 321.1961 & -0.01 & 0.99 & 10.5 & $\mathrm{C} 21 \mathrm{H} 25 \mathrm{O}$ N2 \\
\hline 335.2118 & 335.2118 & 0.03 & 0.33 & 10.5 & $\mathrm{C} 22 \mathrm{H} 27 \mathrm{O} N 2$ \\
\hline 349.2274 & 349.2274 & -0.02 & 0.4 & 10.5 & $\mathrm{C} 23 \mathrm{H} 29 \mathrm{O} \mathrm{N} 2$ \\
\hline 363.2431 & 363.2431 & 0.05 & 0.24 & 10.5 & C24 H31 O N2 \\
\hline 377.2587 & 377.2587 & -0.04 & 0.27 & 10.5 & $\mathrm{C} 25 \mathrm{H} 33 \mathrm{O}$ N2 \\
\hline 391.2744 & 391.2744 & -0.01 & 0.31 & 10.5 & $\mathrm{C} 26 \mathrm{H} 35 \mathrm{O}$ N2 \\
\hline 405.2899 & 405.2900 & -0.09 & 0.31 & 10.5 & $\mathrm{C} 27 \mathrm{H} 37 \mathrm{O} N 2$ \\
\hline 419.3057 & 419.3057 & -0.01 & 0.21 & 10.5 & $\mathrm{C} 28 \mathrm{H} 39 \mathrm{O}$ N2 \\
\hline 575.4934 & 575.4935 & -0.05 & 27.41 & 9.5 & C39 H63 O N2 \\
\hline
\end{tabular}

Table S2 The HR CID MS2 spectrum of 2,4,6-trimethyl tetracos-2,15-dienoic acid-AMPP at m/z 573

\begin{tabular}{|c|c|c|c|c|c|}
\hline $\begin{array}{l}\text { measured } \\
\mathrm{m} / \mathrm{z} \text { (Da) }\end{array}$ & $\begin{array}{r}\text { Theo. Mass } \\
\text { Da }\end{array}$ & $\begin{array}{l}\text { Delta (mmu) } \\
\mathrm{mDa}\end{array}$ & $\begin{array}{l}\text { Relative } \\
\%\end{array}$ & RDB equiv. & Composition \\
\hline 168.0807 & 168.0807 & 0.12 & 6.56 & 8.5 & $\mathrm{C} 12 \mathrm{H} 10 \mathrm{~N}$ \\
\hline 169.0886 & 169.0886 & 0 & 44.96 & 8 & $\mathrm{C} 11 \mathrm{H} 12 \mathrm{~N}$ \\
\hline 183.0917 & 183.0917 & 0.02 & 82.79 & 8.5 & C12 H11 N2 \\
\hline 211.086 & 211.0866 & -0.55 & 1.5 & 9.5 & C13 H11 O N2 \\
\hline 223.1228 & 223.123 & -0.18 & 12.66 & 9.5 & C15 H15 N2 \\
\hline 251.1172 & 251.1179 & -0.74 & 90.31 & 10.5 & C16 H15 O N2 \\
\hline 265.133 & 265.1335 & -0.57 & 12.67 & 10.5 & C17 H17 O N2 \\
\hline 279.1488 & 279.1492 & -0.35 & 100 & 10.5 & C18 H19 O N2 \\
\hline 293.1645 & 293.1648 & -0.33 & 33.99 & 10.5 & $\mathrm{C} 19 \mathrm{H} 21 \mathrm{O} \mathrm{N} 2$ \\
\hline 307.1794 & 307.1805 & -1.14 & 0.33 & 10.5 & $\mathrm{C} 20 \mathrm{H} 23 \mathrm{O} \mathrm{N} 2$ \\
\hline 321.1953 & 321.1961 & -0.83 & 6.77 & 10.5 & $\mathrm{C} 21 \mathrm{H} 25 \mathrm{O} \mathrm{N} 2$ \\
\hline 335.2111 & 335.2118 & -0.73 & 5.78 & 10.5 & $\mathrm{C} 22 \mathrm{H} 27 \mathrm{O} \mathrm{N} 2$ \\
\hline 349.227 & 349.2274 & -0.41 & 4.44 & 10.5 & $\mathrm{C} 23 \mathrm{H} 29 \mathrm{O} \mathrm{N} 2$ \\
\hline 363.2427 & 363.2431 & -0.36 & 4.09 & 10.5 & $\mathrm{C} 24 \mathrm{H} 31 \mathrm{O} N 2$ \\
\hline 377.2588 & 377.2587 & 0.03 & 15.43 & 10.5 & $\mathrm{C} 25 \mathrm{H} 33 \mathrm{O} \mathrm{N} 2$ \\
\hline 391.2745 & 391.2744 & 0.08 & 11.97 & 10.5 & $\mathrm{C} 26 \mathrm{H} 35 \mathrm{O} \mathrm{N} 2$ \\
\hline 405.2902 & 405.29 & 0.12 & 7.99 & 10.5 & $\mathrm{C} 27 \mathrm{H} 37 \mathrm{O}$ N2 \\
\hline 419.3059 & 419.3057 & 0.19 & 11.17 & 10.5 & $\mathrm{C} 28 \mathrm{H} 39 \mathrm{O}$ N2 \\
\hline 433.3216 & 433.3213 & 0.23 & 1.2 & 10.5 & $\mathrm{C} 29 \mathrm{H} 41 \mathrm{O} \mathrm{N} 2$ \\
\hline
\end{tabular}




$\begin{array}{lrlrll}447.3373 & 447.337 & 0.31 & 0.76 & 10.5 & \text { C30 H43 O N2 } \\ 459.3373 & 459.337 & 0.35 & 0.42 & 11.5 & \text { C31 H43 O N2 } \\ 475.3687 & 475.3683 & 0.39 & 3.42 & 10.5 & \text { C32 H47 O N2 } \\ 573.4783 & 573.4778 & 0.47 & 14.17 & 10.5 & \text { C39 H61 O N2 }\end{array}$

Table S3. The HR HCD MS2 spectrum of 2,4-dimethyl tetracosanoic acid-AMPP at m/z 535

\begin{tabular}{|c|c|c|c|c|c|}
\hline $\begin{array}{l}\text { measured } \\
m / z(D a)\end{array}$ & $\begin{array}{r}\text { Theo. Mass } \\
\text { Da }\end{array}$ & $\begin{array}{l}\text { Delta (mmu) } \\
\text { mDa }\end{array}$ & $\begin{array}{l}\text { Relative } \\
\%\end{array}$ & RDB equiv. & Composition \\
\hline 169.0886 & 169.0886 & 0 & 16.85 & 8 & $\mathrm{C} 11 \mathrm{H} 12 \mathrm{~N}$ \\
\hline 183.0917 & 183.0917 & 0.06 & 100 & 8.5 & C12 H11 N2 \\
\hline 211.0861 & 211.0866 & -0.45 & 3.45 & 9.5 & $\mathrm{C} 13 \mathrm{H} 11 \mathrm{O}$ N2 \\
\hline 239.1171 & 239.1179 & -0.81 & 15.82 & 9.5 & C15 H15 O N2 \\
\hline 253.1329 & 253.1335 & -0.65 & 35.02 & 9.5 & $\mathrm{C} 16 \mathrm{H} 17 \mathrm{O}$ N2 \\
\hline 281.1646 & 281.1648 & -0.22 & 5.31 & 9.5 & $\mathrm{C} 18 \mathrm{H} 21 \mathrm{O}$ N2 \\
\hline 295.1803 & 295.1805 & -0.21 & 1.62 & 9.5 & $\mathrm{C} 19 \mathrm{H} 23 \mathrm{O} \mathrm{N} 2$ \\
\hline 309.1952 & 309.1961 & -0.93 & 5.37 & 9.5 & $\mathrm{C} 20 \mathrm{H} 25 \mathrm{O} \mathrm{N} 2$ \\
\hline 323.2112 & 323.2118 & -0.64 & 3.5 & 9.5 & $\mathrm{C} 21 \mathrm{H} 27 \mathrm{O} \mathrm{N} 2$ \\
\hline 337.2269 & 337.2274 & -0.58 & 6.12 & 9.5 & $\mathrm{C} 22 \mathrm{H} 29 \mathrm{O} \mathrm{N} 2$ \\
\hline 351.2428 & 351.2431 & -0.26 & 7.71 & 9.5 & $\mathrm{C} 23 \mathrm{H} 31 \mathrm{O} \mathrm{N} 2$ \\
\hline 365.2586 & 365.2587 & -0.18 & 8 & 9.5 & $\mathrm{C} 24 \mathrm{H} 33 \mathrm{O} \mathrm{N} 2$ \\
\hline 379.2746 & 379.2744 & 0.18 & 7.98 & 9.5 & $\mathrm{C} 25 \mathrm{H} 35 \mathrm{O}$ N2 \\
\hline 393.2903 & 393.29 & 0.26 & 7.04 & 9.5 & $\mathrm{C} 26 \mathrm{H} 37 \mathrm{O} \mathrm{N} 2$ \\
\hline 407.306 & 407.3057 & 0.29 & 6.48 & 9.5 & $\mathrm{C} 27 \mathrm{H} 39 \mathrm{O} \mathrm{N} 2$ \\
\hline 421.3217 & 421.3213 & 0.39 & 5.91 & 9.5 & $\mathrm{C} 28 \mathrm{H} 41 \mathrm{O} \mathrm{N} 2$ \\
\hline 435.3374 & 435.337 & 0.44 & 5.39 & 9.5 & $\mathrm{C} 29 \mathrm{H} 43 \mathrm{O} \mathrm{N} 2$ \\
\hline 449.3531 & 449.3526 & 0.5 & 4.15 & 9.5 & $\mathrm{C} 30 \mathrm{H} 45 \mathrm{O} \mathrm{N} 2$ \\
\hline 463.3688 & 463.3683 & 0.55 & 3.08 & 9.5 & C31 H47 O N2 \\
\hline 477.3845 & 477.3839 & 0.61 & 1.47 & 9.5 & C32 H49 O N2 \\
\hline 491.4003 & 491.3996 & 0.67 & 0.53 & 9.5 & C33 H51 O N2 \\
\hline 505.416 & 505.4152 & 0.79 & 0.45 & 9.5 & C34 H53 O N2 \\
\hline 519.4316 & 519.4309 & 0.72 & 0.22 & 9.5 & C35 H55 O N2 \\
\hline 535.4631 & 535.4622 & 0.88 & 26.33 & 8.5 & C36 H59 O N2 \\
\hline
\end{tabular}

Table S4. The HR HCD MS2 spectrum of 3-hydroxy 2,4,6-trimethyl tetracosanoic acid-AMPP at m/z 593

\begin{tabular}{|c|c|c|c|c|c|}
\hline $\begin{array}{l}\text { measured } \\
\mathrm{m} / \mathrm{z}(\mathrm{Da})\end{array}$ & $\begin{array}{r}\text { Theo. Mass } \\
\text { Da }\end{array}$ & $\begin{array}{l}\text { Delta (mmu) } \\
\text { mDa }\end{array}$ & $\begin{array}{l}\text { Relative } \\
\%\end{array}$ & RDB equiv. & Composition \\
\hline 169.0886 & 169.0886 & 0 & 32.25 & 8.5 & C11 H12 N \\
\hline 185.1074 & 185.1073 & 0.08 & 18.25 & 7.5 & $\mathrm{C} 12 \mathrm{H} 13 \mathrm{~N} 2$ \\
\hline 241.1327 & 241.1335 & -0.84 & 100 & 8.5 & $\mathrm{C} 15 \mathrm{H} 17 \mathrm{O}$ N2 \\
\hline 593.5409 & 593.5404 & 0.5 & 6.46 & 7.5 & $\mathrm{C} 39 \mathrm{H} 65 \mathrm{O} 2 \mathrm{~N} 2$ \\
\hline
\end{tabular}

